\title{
Muscle wasting associated with the long-term use of mTOR inhibitors
}

\author{
BISHAL GYAWALI ${ }^{1}$, TOMOYA SHIMOKATA ${ }^{1}$, KAZUNORI HONDA ${ }^{1}$, CHIHIRO KONDOH $^{2}$, NAOMI HAYASHI ${ }^{1}$, \\ YASUSHI YOSHINO ${ }^{3}$, NAOTO SASSA $^{3}$, YASUYUKI NAKANO ${ }^{2}$, MOMOKAZU GOTOH $^{3}$ and YUICHI ANDO ${ }^{1}$ \\ ${ }^{1}$ Department of Clinical Oncology and Chemotherapy, Nagoya University Hospital, Nagoya, Aichi 466-8560; \\ ${ }^{2}$ Department of Medical Oncology, Japanese Red Cross Nagoya Daiichi Hospital, Nagoya, Aichi 453-8511; \\ ${ }^{3}$ Department of Urology, Nagoya University Graduate School of Medicine, \\ Nagoya University Hospital, Nagoya, Aichi 466-8560, Japan
}

Received May 18, 2016; Accepted August 10, 2016

DOI: $10.3892 / \mathrm{mco} .2016 .1015$

\begin{abstract}
Some targeted therapies alter muscle mass due to interference with pathways of muscle metabolism. The effects of mammalian target of ra pamycin (mTOR) inhibitors on muscle mass have yet to be fully elucidated. In the present study, the computerized tomography (CT) scans of patients receiving mTOR inhibitors for at least 6 months taken at baseline and post-therapy were retrospectively retrieved, and body composition analyses were performed using the software, sliceOmatic version 5.0 (TomoVision, Inc., Magog, QC, Canada). The difference in body composition parameters was evaluated for significance. The time to treatment (TTF) failure was also compared between the sarcopenic and non-sarcopenic patients at the baseline. Of the 75 patients studied, 20 matched the inclusion criteria (including 16 males). The mean duration between the CT scans was $14.4 \pm 2.0$ months. A total of $12(60 \%)$ patients were sarcopenic at the baseline, whereas three more $(75 \%$ in total) became sarcopenic following treatment. The use of mTOR inhibitors significantly decreased the skeletal muscle area $(\mathrm{P}=0.011)$ and lean body mass $(\mathrm{P}=0.007)$, although it had no effect on adipose tissue $(\mathrm{P}=0.163)$ or body weight $(\mathrm{P}=0.262)$. The rate of skeletal muscle wasting was $2.6 \mathrm{~cm}^{2} / \mathrm{m}^{2}$, or $2.3 \mathrm{~kg}$ in 6 months. The TTF did not differ between sarcopenic and non-sarcopenic patients, and was not significantly associated with any other parameter. To the best of our knowledge, this is the first study to demonstrate that the long-term use of mTOR inhibitors induces a marked loss of muscle mass. Due to the predictive
\end{abstract}

Correspondence to: Dr Bishal Gyawali, Department of Clinical Oncology and Chemotherapy, Nagoya University Hospital, 65 Tsurumai-cho, Showa-ku, Nagoya, Aichi 466-8550, Japan E-mail: bg.bishalgyawali@gmail.com

Key words: mammalian target of rapamycin, mTOR inhibitors, skeletal muscle index, skeletal muscle mass, sarcopenia, cancer cachexia and prognostic role of sarcopenia in cancer patients, these findings may have important clinical implications.

\section{Introduction}

Low muscle mass, also known as sarcopenia (1), is of interest and importance in oncology due to its known association with prognosis and toxicities in a variety of cancer types (2-5) and cancer drugs (6-9). Sarcopenia forms an important component in the definition of cancer cachexia (10).

The phosphoinositide 3-kinase (PI3K)/protein kinase B (AKT)/mammalian target of rapamycin (mTOR) pathway has a key role in activating skeletal muscle synthesis via various stimuli $(11,12)$. According to in vivo experiments, upregulation of this pathway leads to muscle hypertrophy, whereas the genetic blockade of this pathway blocks hypertrophy $(11,12)$. Therefore, inhibition of this pathway might lead to loss of muscle mass in patients who receive an mTOR inhibitor for cancer treatment. In fact, the finding of sarcopenia has already been demonstrated with the use of the multi-kinase inhibitor, sorafenib, presumably due to downstream suppression of PI3K, AKT and mTOR (13). Surprisingly, however, the muscle mass increased on treatment with the mitogen-activated extracellular signal-related kinase kinase inhibitor, selumetinib (14), and the tyrosine kinase inhibitor, vandetanib (9). To the best of our knowledge, no reports have been previously published on the significant changes in muscle area with the long-term use of mTOR inhibitors, such as everolimus and temsirolimus. As mTOR inhibitors are now being widely used for a wide variety of conditions, including renal cell cancer, breast cancer and pancreatic neuroendocrine tumors among others, information on whether the use of mTOR inhibitors induces sarcopenia is gaining importance. Hence, the aim of the present study was to examine the effect of mTOR inhibitors on muscle mass.

The primary objective of the present study was to assess the change in muscle mass during the long-term (at least 6 months) use of mTOR inhibitors. The secondary objectives were to assess the other parameters of body composition, as well as to investigate whether the time to treatment failure (TTF) differed between the sarcopenic and non-sarcopenic patients at the baseline. Although it is now known that the 
assessment of body weight or the body mass index (BMI) is not able to reliably predict the loss of muscle mass, it has yet to be elucidated whether serum albumin and serum C-reactive protein (CRP), markers of nutrition and inflammation, are able to be used as predictors of the alteration in muscle mass. Hence, the levels of serum albumin and CRP as markers of cancer cachexia were also assessed in the present study.

\section{Materials and methods}

Retrospective analysis. This is a retrospective study performed in two institutions of Nagoya, Japan: The Nagoya University Hospital and the Japanese Red Cross Nagoya Daiichi Hospital. The eligibility criteria for inclusion in the present study were: Patients who had taken everolimus or temsirolimus as a single drug therapy for at least 6 months (dose-skipping due to side effects allowed) and who had computerized tomography (CT) scans of the abdomen at the level of the third lumbar vertebrae (L3) available at within \pm 1 month from the date of starting the drug therapy, and within 1 month of having received the drug for at least 6 months. Data from between June 2010 and October 2015 were accessed. Approval was received from the Institutional Review Board of the two hospitals prior to performing the study.

Body composition measurement. Body weight and height were obtained from the medical chart. BMI was measured using the formula: Weight in $\mathrm{kg} /(\text { height in } \mathrm{m})^{2}$. CT scans of the abdomen at the baseline and following at least 6 months of therapy were obtained from the electronic database. The L3 level slice was identified, body composition parameters were measured in two slices of the CT scan starting from the L3 level downwards, and averages of the values of the two were determined, as previously described $(13,15)$. Body composition was measured using the software sliceOmatic version 5.0 (TomoVision, Inc., Magog, QC, Canada). The L3 level was chosen as it is a validated marker for body composition analysis, including skeletal muscle and adipose tissue $(16,17)$. Subcutaneous adipose tissue (SAT), visceral adipose tissue (VAT) and skeletal muscle tissue (SMT) at the L3 level were identified using the CT Hounsfield units of: -190 to -30 for SAT; -150 to -50 for VAT; and -29 to +150 for SMT, as previously described (13). The muscles identified in the $\mathrm{L} 3$ region were psoas, paraspinal muscles (erector spinae, quadratus lumborum) and abdominal wall muscles (transversus abdominis, external and internal obliques, rectus abdominis). The areas $\left(\right.$ in $\mathrm{cm}^{2}$ ) of the SAT, VAT and SMT were provided by the software. Total adipose tissue (TAT) was calculated as SAT + VAT. The values of the areas obtained for SAT, VAT, TAT and SMT were subsequently adjusted for body height by dividing the area by the square of the body height $\left(\mathrm{cm}^{2} / \mathrm{m}^{2}\right)$. The SMT area thus adjusted for body height is known as the L3 skeletal muscle index (SMI), and this is a validated parameter for the assessment of sarcopenia $(10,15)$. Lean body mass (LBM) was also calculated using the formula: $\mathrm{LBM}(\mathrm{kg})=0.30 \mathrm{x}\left(\mathrm{SMT}\right.$ area at L3 in CT in $\left.\mathrm{cm}^{2}\right)+6.06(16)$ Sarcopenia was defined as SMI $\leq 38.5 \mathrm{~cm}^{2} / \mathrm{m}^{2}$ for females and $\leq 52.4 \mathrm{~cm}^{2} / \mathrm{m}^{2}$ for males, using the definition of Prado et al (15) to study the incidence of sarcopenia in our population. Since this definition has not been validated for the Japanese popula- tion, the gender-specific median SMI of our study population was used instead as the cut-off for sarcopenia.

Serum albumin and CRP values were also obtained at dates corresponding to the $\mathrm{CT}$ scans to investigate their potential role in predicting the loss of muscle mass.

Statistical analysis. The mean values for various parameters were calculated at the baseline and following the use of the mTOR inhibitor, and the differences were examined for statistical significance using a paired $t$-test. Furthermore, to account for the variation in the duration of scan intervals, as well as to find the rate of the change in body composition parameters, the differences in body parameters were converted in terms of 180 days ( 6 months) by using the formula: (Change in parameter/no. of days between scans) x180; this difference was assessed for significance using a one-sample $t$-test.

The association of each body parameter with a change in L3 index was evaluated with bivariate analysis using Pearson's correlation coefficient, and the significance was examined using a two-tailed test. TTF was defined as the time from the start of taking the mTOR inhibitor to the time of stoppage of drug use, due to adverse events, disease progression or mortality, depending on whichever occurred first. The TTF was calculated and compared between sarcopenic and non-sarcopenic patients at the baseline using the Kaplan-Meier method and the log-rank test. The correlations between TTF and various parameters were examined using univariable Cox regression analyses. All P-values were two-sided, and $\mathrm{P}<0.05$ was taken to indicate a statistically significant value. All the statistical analyses were performed using SPSS software, version 22.0 (IBM SPSS, Armonk, NY, USA).

\section{Results}

Among a total of 75 patients who had received mTOR inhibitor therapy during the specified period, 20 met the inclusion criteria and were included in the present study (Fig. 1). Pediatric patients were excluded, since their skeletal muscle mass cannot be compared with that of adults, whereas patients with angiomyolipoma were also excluded since their skeletal muscles were entirely compressed by the excessive volume of the tumor at the L3 level, which rendered any muscle area assessment using CT scans obsolete. Patients with breast cancer on everolimus + hormone therapy, and patients with pancreatic neuroendocrine tumors on everolimus + octreotide, were excluded as the influence of everolimus alone on body composition could not be definitively ascertained.

The mean duration of mTOR inhibitor use was 14.1 \pm 2.1 months, and the mean duration between the first and final CT scans was $14.4 \pm 2.0$ months. All patients had metastatic renal cell carcinoma, with the exception of two who had metastatic pancreatic neuroendocrine tumors. None of the patients were obese; by contrast, five of them (25\%) were underweight $\left(\mathrm{BMI}<18.5 \mathrm{~kg} / \mathrm{m}^{2}\right.$; Table I).

Effect of the long-term use of mTOR inhibitors on body composition parameters and TTF. A total of 16 patients (80\%) suffered a loss of the SMT area, SMI and LBM following at least 6 months of drug use compared with the baseline. A gain in body weight was observed in nine patients, although seven 
Table I. Patient characteristics.

Parameter Value Percentage (\%)

Total no. of patients

Drug use

Everolimus alone

Temsirolimus alone

Disease

Renal cell carcinoma

Pancreatic neuroendocrine tumor

Gender

Male

Female

Age in years (median)

Duration of therapy in months (mean)

CT interval in months (mean)

Baseline BMI category $\left(\mathrm{kg} / \mathrm{m}^{2}\right)$

Normal (18.5-24.9)

Underweight $(<18.5)$

Overweight (25.0-29.9)

Patients with baseline sarcopenia ${ }^{a}$

Patients with sarcopenia post-treatment ${ }^{\mathrm{a}}$

Median baseline L3 index (in $\mathrm{cm}^{2} / \mathrm{m}^{2}$ )
20

12

60

40

18

90

10

16

80

4

$65.5(45-83)$

$14.1 \pm 2.1$

$14.4 \pm 2.0$

13

65

$5-25$

210

12 (underweight $=4$; normal BMI $=8$ ) 60

15

75

50.5 for males

30.5 for females

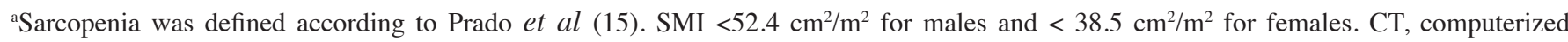
tomography; BMI, body mass index; SMI, skeletal muscle index; L3, level of the third lumbar vertebrae.

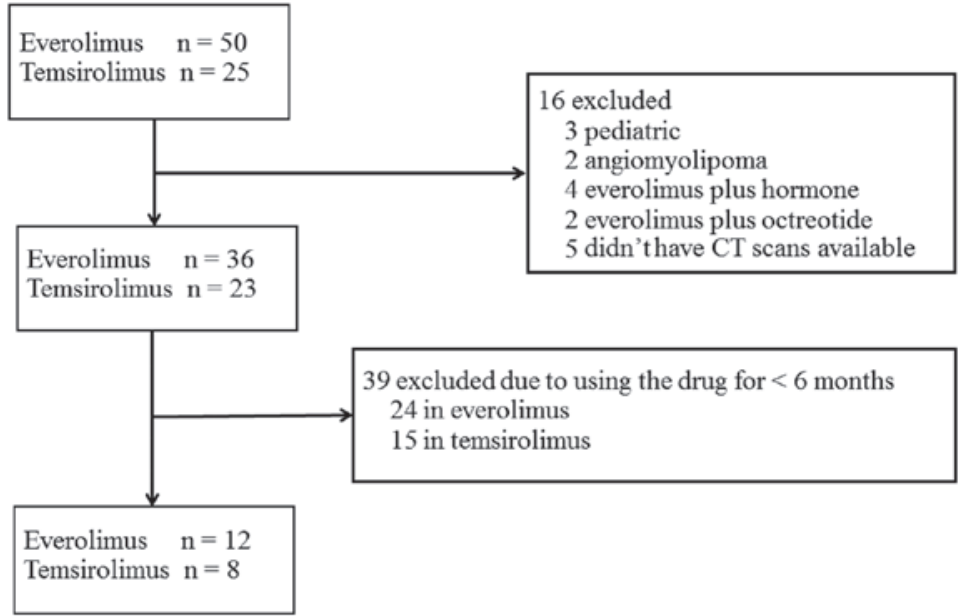

Figure 1. A flow diagram showing patient enrollment in the present study.

of them suffered loss of muscle despite gaining body weight. The number of sarcopenic patients increased post-therapy (75\%) compared with the baseline (60\%; Table I).

The decrease in body weight was marginal, and not significant. The indices for SAT, VAT and TAT increased post-treatment compared with the baseline, although this increment was not statistically significant (Table II). The parameters SMT area, SMI and LBM all decreased signifi- cantly following treatment: For the SMT area, the mean decrease was $12.6 \mathrm{~cm}^{2}[\mathrm{P}=0.011,95 \%$ confidence interval (CI) for decrease in mean area, 3.3 to 22.0], for SMI, the mean decrease was $6.3 \mathrm{~cm}^{2} / \mathrm{m}^{2}(\mathrm{P}=0.022 ; 95 \% \mathrm{CI}$ for decrease in mean index, 1.0 to 11.7) and for LBM, the mean decrease was $4.1 \mathrm{~kg},(\mathrm{P}=0.007,95 \% \mathrm{CI}$ for decrease in mean mass, 1.3 to 6.9). The rate of decrease in SMI was $2.6 \mathrm{~cm}^{2} / \mathrm{m}^{2}(\mathrm{P}=0.022)$ in 6 months (180 days). LBM decreased by $2.3 \mathrm{~kg}(\mathrm{P}=0.016)$ 
Table II. Effect of long-term mTOR inhibitors on the mean body composition parameters.

\begin{tabular}{|c|c|c|c|c|c|}
\hline Parameter & $\begin{array}{l}\text { Prior to the } \\
\text { start of the } \\
\text { treatment }\end{array}$ & $\begin{array}{l}\text { Following at } \\
\text { least } 6 \text { months } \\
\text { of treatment }\end{array}$ & Difference & $\begin{array}{c}95 \% \\
\text { confidence } \\
\text { interval }\end{array}$ & P-value \\
\hline Body weight (kg) & 55.5 & 54.4 & -1.0 & -2.9 to 0.8 & 0.262 \\
\hline SAT index $\left(\mathrm{cm}^{2} / \mathrm{m}^{2}\right)$ & 35.1 & 36.4 & 1.3 & -6.3 to 8.8 & 0.722 \\
\hline VAT index $\left(\mathrm{cm}^{2} / \mathrm{m}^{2}\right)$ & 31.5 & 43.8 & 12.3 & -0.2 to 24.7 & 0.053 \\
\hline TAT index $\left(\mathrm{cm}^{2} / \mathrm{m}^{2}\right)$ & 66.6 & 80.2 & 13.6 & -6.0 to 33.1 & 0.163 \\
\hline SMT area at $\left(\mathrm{cm}^{2}\right)$ & 137.3 & 124.6 & -12.6 & -22.0 to -3.3 & $0.011^{\mathrm{a}}$ \\
\hline $\operatorname{SMI}\left(\mathrm{cm}^{2} / \mathrm{m}^{2}\right)$ & 50.2 & 43.8 & -6.3 & -11.7 to -1.0 & $0.022^{\mathrm{a}}$ \\
\hline Lean body mass (kg) & 47.2 & 43.1 & -4.1 & -6.9 to -1.3 & $0.007^{\mathrm{a}}$ \\
\hline Serum albumin (g/dl) & 3.7 & 3.5 & -0.3 & -0.6 to 0.0 & 0.091 \\
\hline $\mathrm{CRP}(\mathrm{mg} / \mathrm{dl})$ & 2.8 & 5.3 & 2.5 & -0.6 to 5.5 & 0.105 \\
\hline \multicolumn{6}{|c|}{ Mean of rate of change per 180 days } \\
\hline $\operatorname{SMI}\left(\mathrm{cm}^{2} / \mathrm{m}^{2}\right)$ & & & -2.6 & -4.9 to -0.4 & $0.022^{\mathrm{a}}$ \\
\hline Lean body mass $(\mathrm{kg})$ & & & -2.3 & -4.2 to -0.5 & $0.016^{\mathrm{a}}$ \\
\hline
\end{tabular}

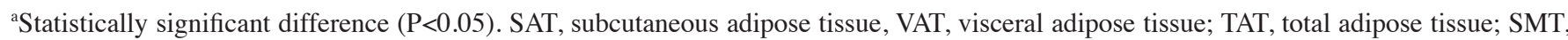
skeletal muscle tissue; SMI, skeletal muscle index; CRP, C-reactive protein.

Table III. Correlation of the changes in L3 index with various parameters.

\begin{tabular}{lcc}
\hline & $\begin{array}{c}\text { Pearson correlation } \\
\text { coefficient }\end{array}$ & P-value \\
\hline Baseline BMI & -0.2 & 0.292 \\
Baseline SMI & 0.5 & 0.023 \\
Duration of therapy & -0.04 & 0.855 \\
Age & 0.15 & 0.539 \\
Gender & 0.260 & 0.268 \\
Change in: & & \\
BW & 0.1 & 0.721 \\
Serum albumin & -0.1 & 0.674 \\
CRP & 0.3 & 0.229 \\
\hline
\end{tabular}

L3, level of the third lumbar vertebrae; BMI, body mass index; SMI, skeletal muscle index; BW, body weight; CRP, C-reactive protein.

in 6 months. The level of serum albumin decreased, and CRP increased, following the drug use compared with the baseline, although the differences were not significant.

Association of muscle loss with various parameters. The extent of the decrease in SMI was examined quantitatively in terms of correlation with various parameters, as shown in Table III. The only significant association was with the baseline SMI; that is, the lower the baseline SMI, the less the decrease in muscle mass. However, the changes in L3 index did not correlate significantly with the duration of drug use.

Association of TTF with SMI. Taking the median SMI as the cut-off value based on gender, the median TTF was longer

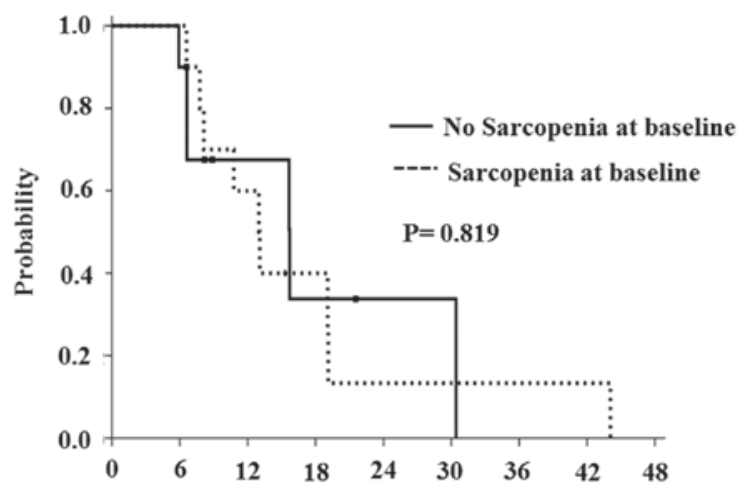

\begin{tabular}{lccccccccc} 
Number at Risk & \multicolumn{7}{c}{ Time to Treatment Failure } \\
\hline Sarcopenia & 10 & 10 & 6 & 3 & 1 & 1 & 1 & 1 & 0 \\
No sarcopenia & 10 & 9 & 4 & 2 & 1 & 1 & 0 & 0 & 0 \\
\hline
\end{tabular}

Figure 2. Comparison between the time to treatment failure in patients who had sarcopenia at the baseline and those who did not have sarcopenia at baseline. The gender-specific median skeletal muscle index was taken as the cut-off for defining sarcopenia.

in the non-sarcopenic arm (15.7 months) compared with the sarcopenic arm (12.9 months), although the difference was not significant ( $\mathrm{P}=0.819$; Fig. 2). Among the various parameters studied, no significant correlation of any of them was observed with TTF.

\section{Discussion}

To the best of our knowledge, this is the first study to demonstrate that the long-term use of mTOR inhibitors significantly decreases the muscle quantity, without affecting body weight and adipose tissue. The present study has also shown that the amount of the decrease in muscle mass was associated with the baseline muscle mass. TTF was not associated with the sarcopenia status of the patients. 
The chronological assessment and interpretation of alterations in muscle mass in cancer patients is problematical, since loss of muscle mass is an important part of cancer cachexia (10). Hence, it is difficult to accurately determine whether the change in muscle mass observed is due to cancer cachexia or due to the treatment being followed (18). Therefore, the use of the drug for at least 6 months was taken as an inclusion criterion so that the observed alteration in muscle mass could be reliably ascribed to the drug, and not simply be a component of cancer cachexia. Since cancer cachexia rapidly progresses in patients without treatment response, any muscle loss in those patients is likely to be due to the disease, and not the drug. Furthermore, drug-induced muscle loss at 6 months was previously confirmed in a placebo-controlled trial of sorafenib (13).

Unlike other studies that have usually assessed body composition change over 3 months $(9,14)$, at least 6 months of drug use was featured in the present study as an inclusion criterion to increase the reliability that the observed differences were due to the drug. One previous study evaluating the effect of temsirolimus on body composition did not demonstrate a statistically significant decrease in muscle area, unlike our study (19). One plausible explanation to account for this is that the mean time between the two evaluated CT scans was only 9 weeks in that study, compared with 14 months in the present study. To the best of our knowledge, this is the longest mean duration between the CT scans for the evaluation of body composition change with drug use. Loss of body weight is an important component of cancer cachexia (10). Since there was no significant loss of body weight, the loss of muscle mass observed in the present study was most likely due to the drug, and not a part of cancer cachexia.

Seven patients in the present study suffered a loss of muscle area, despite gaining weight. Decreases in SMI were not correlated with losses in body weight. A quarter $(25 \%)$ of the patients had a low BMI, whereas $60 \%$ of the patients were sarcopenic at the baseline. Taken together, the present study confirms that the assessment of sarcopenia cannot (and should not) be substituted with body weight or BMI measurement alone. Sarcopenia, a predictor of worse prognosis, may remain unnoticed, particularly in patients with normal body weight or BMI. This is particularly important in the Western world, where the percentage of obese patients is high. Therefore, increases in body weight may not always be reassuring, since numerous patients could continue to lose muscle mass in spite of gaining body weight.

The exact mechanisms underpinning the loss of muscle mass with the use of mTOR inhibitors warrant further investigation. Activation of the PI3K-AKT-mTOR pathway is essential for muscle protein synthesis. It has been reported that the activation of the AKT/mTOR pathway and its downstream targets is essential for regulating skeletal muscle fiber size, and that the activation of the AKT/mTOR pathway may oppose the muscle atrophy that is induced by disuse (11). Genetic activation of the AKT/mTOR pathway was sufficient to cause hypertrophy and prevent atrophy in vivo, whereas genetic blockade of this pathway blocked hypertrophy in vivo (12). It has also been observed that the increase in muscle mass in response to nutrition or exercise occurs in parallel with the activation of mammalian target of rapamycin complex 1 (mTORC1) (20). In the present study, no significant association of skeletal muscle loss with the gain or loss of adipose tissue was observed. This substantiates the definition of cancer cachexia as muscle loss with or without the loss of adipose tissue (10).

A majority of the sarcopenia studies in an oncological setting have used Prado's cut-off $\left(<38.5 \mathrm{~cm}^{2} / \mathrm{m}^{2}\right.$ for females and $<52.4 \mathrm{~cm}^{2} / \mathrm{m}^{2}$ for males) for segregating sarcopenic from non-sarcopenic patients (15). However, in the study of Prado et al (15), the cut-off value was based on the Canadian population, and it may not be used for a Japanese population such as the one featured in the present study, a fact that has already been acknowledged by other members of the same group that originally provided this cut-off (21). Thus, in the present study, the gender-specific median SMI of our own population was used to evaluate the TTF, as SMI cut-off data for Asian populations are not available.

One interesting finding in the present study was the significant correlation between the change in SMI and the baseline SMI, namely, that patients who have a low muscle mass at the baseline do not experience as much loss of muscle mass during treatment as do patients who have greater muscle mass at the baseline. Given the important role of the mTOR pathway in muscle synthesis, patients who have low muscle mass at the baseline may also have low levels of mTORC1 receptors at the baseline, compared with patients with comparatively more muscle mass. Thus, the effect of mTOR inhibition may not be as pronounced in patients with low muscle mass at the baseline. However, this is only a hypothesis, and further confirmatory studies are required. Although not significantly so, the decrease in serum albumin and the increase in the level of CRP observed in the present study were expected findings, as serum albumin and CRP are markers of inflammation, and sarcopenia is known to be influenced by inflammation.

Unlike other studies, the present study was not able to demonstrate significantly shorter TTF of sarcopenic patients compared with those without sarcopenia. This could be due to the exclusion of patients with rapid disease progression in our study, as only those patients with at least 6 months of continuous therapy with mTOR inhibitors were included. Alternatively, for patients who received mTOR inhibitor therapy for more than 6 months, sarcopenia may not be a poor prognostic factor, unlike the situation with other drugs. Neither was studying the effects of sarcopenic obesity possible, as none of our patients were obese. The amount of, or the change in, the VAT or TAT values had no impact on TTF. However, due to the small sample size, conclusions regarding TTF or survival may not be drawn from the present study.

The retrospective nature and lack of a control group are the major limitations of the present study. The use of a placebo control is impossible in a retrospective study, and it would also be unethical, with the exception of clinical trials. Non-responders (i.e. who took the drug for $<6$ months) could have formed a possible control group, although as mentioned above, muscle loss in this group would be more likely to be due to cancer cachexia, and not the drug, thereby rendering comparisons obsolete. The small sample size is another limitation of the present study. However, a number of previous studies on sarcopenia in an oncological setting have usually had a small sample size [e.g. 16 patients in the study of 
temsirolimus (19), and 40 in the study of sorafenib among patients with hepatocellular carcinoma (7)].

In conclusion, the present study has revealed the sarcopenic effect of long-term mTOR inhibitor use, and the importance of making an assessment of muscle mass independently of body weight or the measurement of BMI. Due to the predictive and prognostic role of sarcopenia in cancer patients, this finding may have important therapeutic implications. Indeed, a prospective study to confirm these findings is already in place (UMIN Registration no: UMIN000019477).

\section{Acknowledgements}

We would like to thank Mrs. Yuka Murasaki for administrative support, and Dr Kenta Murotani, for assistance with the statistical analysis. This work was partly supported by JSPS KAKENHI Grant no. 2646026 to Dr. Ando, and research grants from the Kobayashi International Scholarship Foundation to Dr. Gyawali and Dr. Shimokata.

\section{References}

1. Baumgartner RN, Koehler KM, Gallagher D, Romero L, Heymsfield SB, Ross RR, Garry PJ and Lindeman RD: Epidemiology of sarcopenia among the elderly in New Mexico. Am J Epidemiol 147: 755-763, 1998.

2. Prado CM, Baracos VE, McCargar LJ, Reiman T, Mourtzakis M, Tonkin K, Mackey JR, Koski S, Pituskin E and Sawyer MB: Sarcopenia as a determinant of chemotherapy toxicity and time to tumor progression in metastatic breast cancer patients receiving capecitabine treatment. Clin Cancer Res 15: 2920-2926, 2009.

3. van Vledder MG, Levolger S, Ayez N, Verhoef C, Tran TC and Ijzermans JN: Body composition and outcome in patients undergoing resection of colorectal liver metastases. Br J Surg 99 550-557, 2012.

4. Tan BH, Birdsell LA, Martin L, Baracos VE and Fearon KC: Sarcopenia in an overweight or obese patient is an adverse prognostic factor in pancreatic cancer. Clin Cancer Res 15: 6973-6979, 2009.

5. Grossberg AJ, Chamchod S, Fuller CD, Mohamed AS, Heukelom J, Eichelberger H, Kantor ME, Hutcheson KA, Gunn GB, Garden AS, et al: Association of body composition with survival and locoregional control of radiotherapy-treated head and neck squamous cell carcinoma. JAMA Oncol 2: 782-789, 2016.

6. Antoun S, Baracos VE, Birdsell L, Escudier B and Sawyer MB: Low body mass index and sarcopenia associated with dose-limiting toxicity of sorafenib in patients with renal cell carcinoma. Ann Oncol 21: 1594-1598, 2010.

7. Mir O, Coriat R, Blanchet B, Durand JP, Boudou-Rouquette P, Michels J, Ropert S, Vidal M, Pol S, Chaussade S and Goldwasser F: Sarcopenia predicts early dose-limiting toxicities and pharmacokinetics of sorafenib in patients with hepatocellular carcinoma. PLoS One 7: e37563, 2012.
8. Huillard O, Mir O, Peyromaure M, Tlemsani C, Giroux J, Boudou-Rouquette P, Ropert S, Delongchamps NB, Zerbib M and Goldwasser F: Sarcopenia and body mass index predict sunitinib-induced early dose-limiting toxicities in renal cancer patients. Br J Cancer 108: 1034-1041, 2013.

9. Massicotte MH, Borget I, Broutin S, Baracos VE, Leboulleux S, Baudin E, Paci A, Deroussent A, Schlumberger M and Antoun S: Body composition variation and impact of low skeletal muscle mass in patients with advanced medullary thyroid carcinoma treated with vandetanib: results from a placebo-controlled study. J Clin Endocrinol Metab 98: 2401-2408, 2013.

10. Fearon K, Strasser F, Anker SD, Bosaeus I, BrueraE, FainsingerRL, Jatoi A, Loprinzi C, MacDonald N, Mantovani G, et al: Definition and classification of cancer cachexia: An international consensus. Lancet Oncol 12: 489-495, 2011.

11. Bodine SC, Stitt TN, Gonzalez M, Kline WO, Stover GL, Bauerlein R, Zlotchenko E, Scrimgeour A, Lawrence JC, Glass DJ and Yancopoulos GD: Akt/mTOR pathway is a crucial regulator of skeletal muscle hypertrophy and can prevent muscle atrophy in vivo. Nat Cell Biol 3: 1014-1019, 2001.

12. Edinger AL and Thompson CB: Akt maintains cell size and survival by increasing mTOR-dependent nutrient uptake. Mol Biol Cell 13: 2276-2288, 2002.

13. Antoun S, Birdsell L, Sawyer MB, Venner P, Escudier B and Baracos VE: Association of skeletal muscle wasting with treatment with sorafenib in patients with advanced renal cell carcinoma: Results from a placebo-controlled study. J Clin Oncol 28: 1054-1060, 2010.

14. Prado CM, Bekaii-Saab T, Doyle LA, Shrestha S, Ghosh S, Baracos VE and Sawyer MB: Skeletal muscle anabolism is a side effect of therapy with the MEK inhibitor: Selumetinib in patients with cholangiocarcinoma. Br J Cancer 106: 1583-1586, 2012.

15. Prado CM, Lieffers JR, McCargar LJ, Reiman T, Sawyer MB, Martin L and Baracos VE: Prevalence and clinical implications of sarcopenic obesity in patients with solid tumours of the respiratory and gastrointestinal tracts: A population-based study. Lancet Oncol 9: 629-635, 2008.

16. Mourtzakis M, Prado CM, Lieffers JR, Reiman T, McCargar LJ and Baracos VE: A practical and precise approach to quantification of body composition in cancer patients using computed tomography images acquired during routine care. Appl Physiol Nutr Metab 33: 997-1006, 2008.

17. Shen W, Punyanitya M, Wang Z, Gallagher D, St-Onge MP, Albu J, Heymsfield SB and Heshka S: Total body skeletal muscle and adipose tissue volumes: Estimation from a single abdominal cross-sectional image. J Appl Physiol (1985) 97: 2333-2338, 2004.

18. Fearon K, Arends J and Baracos V: Understanding the mechanisms and treatment options in cancer cachexia. Nat Rev Clin Oncol 10: 90-99, 2013.

19. Veasey-Rodrigues H, Parsons HA, Janku F, Naing A, Wheler JJ, Tsimberidou AM and Kurzrock R: A pilot study of temsirolimus and body composition. J Cachexia Sarcopenia Muscle 4: 259-265, 2013.

20. Adegoke OA, Abdullahi A and Tavajohi-Fini P: mTORC1 and the regulation of skeletal muscle anabolism and mass. Appl Physiol Nutr Metab 37: 395-406, 2012.

21. Martin L, Birdsell L, Macdonald N, Reiman T, Clandinin MT, McCargar LJ, Murphy R, Ghosh S, Sawyer MB and Baracos VE: Cancer cachexia in the age of obesity: Skeletal muscle depletion is a powerful prognostic factor, independent of body mass index. J Clin Oncol 31: 1539-1547, 2013. 